\title{
ON SOME NEW GENERALIZATIONS OF CERTAIN GAMIDOV INTEGRAL INEQUALITIES IN TWO INDEPENDENT VARIABLES AND THEIR APPLICATIONS
}

\author{
Khaled Boukerrioua, Dallel Diabi and Brahim Kilani
}

\begin{abstract}
The aim of this paper is to establish some new nonlinear Gamidov integral inequalities in two independent variables which can give the explicit bounds on unknown functions. To show the feasibility of the obtained inequalities, some illustrative examples are also introduced.
\end{abstract}

Keywords: Integral equation, mean value Theorem, Gamidov integral inequality.

\section{Introduction}

The integral inequalities which provide explicit bounds on unknown functions play an important role in the development of the theory of differential and integral equations. For instance, see $[1-19]$ and the references given therein. During the past few years, an enormous amount of effort has been devoted to the discovery of new types of inequalities and their applications in various branches of ordinary and partial differential and integral equations.

In [8], Sh.G.Gamidov, while studying the boundary value problem for higher order differential equations, initiated the study of obtaining explicit upper bounds on the integral inequalities of the forms

$$
u(t) \leq c+\int_{a}^{t} f(s) u(s) d s+\int_{a}^{b} g(s) u(s) d s,
$$

for $t \in[a, b]$, under some suitable conditions on the functions involved in (1.1).

Pachpatte obtained the following interesting explicit bounds on certain integral inequalities which appear in [14] :

Received June 07, 2017; accepted July 21, 2018

2010 Mathematics Subject Classification. 26D15, 26D20, 39A12 


$$
u(t) \leq a(t)+\int_{a}^{t} f(t, s) u(s) d s+\int_{a}^{b} g(s) u(s) d s .
$$

Very recently, K. Cheng, C. Guo in [5] discussed the following general version in two independent variables:

$$
u(x, y) \leq a(x, y)+b(x, y) \int_{0}^{x} \int_{0}^{y} f(s, t) u(s, t) d s d t+c(x, y) \int_{0}^{M} \int_{0}^{N} g(s, t) u(s, t) d s d t,
$$

for $(x, y) \in[0, M] \times[0, N]$.

Motivated by the results above and the inequalities obtained in $[5,8,10,14]$, we give a generalization of nonlinear Gamidov integral inequalities in two independent variables which can be used as a tool to study the boundedness of solutions of integral equations. Some applications are also given to illustrate the usefulness of some of our results.

Before establishing our main results, we need the following lemmas.

Lemma 1.1. [5] Assume $u(x, y), a(x, y), c(x, y), g(x, y) \in C([0, M] \times[0, N],[0, \infty))$ and

$$
u(x, y) \leq a(x, y)+c(x, y) \int_{0}^{M} \int_{0}^{N} u(s, t) g(s, t) d s d t,
$$

for $(x, y) \in[0, M] \times[0, N]$. If $\int_{0}^{M} \int_{0}^{N} c(s, t) g(s, t) d s d t<1$, then the following explicit estimate

$$
u(x, y) \leq a(x, y)+\frac{c(x, y) \int_{0}^{M} \int_{0}^{N} a(s, t) g(s, t) d s d t}{1-\int_{0}^{M} \int_{0}^{N} c(s, t) g(s, t) d s d t},
$$

holds for $(x, y) \in[0, M] \times[0, N]$.

Lemma 1.2. [9] Assume that $a \geq 0, p \geq q \geq 0$ and $p \neq 0$, then

$$
a^{\frac{q}{p}} \leq \frac{q}{p} K^{\frac{q-p}{p}} a+\frac{p-q}{p} K^{\frac{q}{p}},
$$

for any $K>0$.

\section{Main Result}

In what follows, $\mathbb{R}$ denotes the set of real numbers $\mathbb{R}_{+}=[0, \infty), I_{1}=[0, M]$, and $I_{2}=[0, N]$ are given subsets of $\mathbb{R}$. Let $\Delta=I_{1} \times I_{2}, C(U, V)$ denotes the collection of continuous functions from $U$ to $V$. Now let us give the main results of this paper. 
Lemma 2.1. Asssume that $u(x, y), a(x, y), c(x, y), g(x, y) \in C\left(\Delta, \mathbb{R}_{+}\right)$and $n$ : $\mathbb{R}_{+} \rightarrow \mathbb{R}_{+}$a differentiable increasing function on $] 0,+\infty[$ with the continuous nonincreasing first derivative $n^{\prime}$ on $] 0,+\infty[$. If

$$
u(x, y) \leq a(x, y)+c(x, y) \int_{0}^{M} \int_{0}^{N} g(s, t) n(u(s, t)) d s d t
$$

then the following explicit estimate

$$
u(x, y) \leq a(x, y)+\frac{c(x, y) \int_{0}^{M} \int_{0}^{N} g(s, t) n(a(s, t)) d s d t}{1-\int_{0}^{M} \int_{0}^{N} c(s, t) g(s, t) n^{\prime}(a(s, t)) d s d t}
$$

holds for $(x, y) \in \Delta$, provided that

$$
\int_{0}^{M} \int_{0}^{N} c(s, t) g(s, t) n^{\prime}(a(s, t)) d s d t<1 .
$$

Proof. Obviously, $\int_{0}^{M} \int_{0}^{N} g(s, t) n(u(s, t)) d s d t$ is a constant.

Letting

$$
\Omega=\int_{0}^{M} \int_{0}^{N} g(s, t) n(u(s, t)) d s d t
$$

from (2.1), we have

$$
u(x, y) \leq a(x, y)+c(x, y) \Omega .
$$

Since $n$ is increasing on $] 0,+\infty[$, then

$$
n(u(x, y)) \leq n(a(x, y)+c(x, y) \Omega) .
$$

Applying the mean value Theorem for the function $n$, then for every $x_{1} \geq y_{1}>0$ there exists $c \in] y_{1}, x_{1}[$ such that

$$
n\left(x_{1}\right)-n\left(y_{1}\right)=n^{\prime}(c)\left(x_{1}-y_{1}\right) \leq n^{\prime}\left(y_{1}\right)\left(x_{1}-y_{1}\right)
$$

Which gives

$$
n(u(x, y)) \leq n^{\prime}(a(x, y)) c(x, y) \Omega+n(a(x, y)),
$$

taking into account that $g(x, y)$ is positive, then 


$$
g(x, y) n(u(x, y)) \leq g(x, y) n^{\prime}(a(x, y)) c(x, y) \Omega+g(x, y) n(a(x, y)) .
$$

Integrating both sides of (2.9) on $\Delta$, we obtain

$$
\begin{aligned}
\Omega & =\int_{0}^{M} \int_{0}^{N} g(s, t) n(u(s, t)) d s d t \\
& \leq \Omega \int_{0}^{M} \int_{0}^{N} c(s, t) g(s, t) n^{\prime}(a(s, t)) d s d t \\
& +\int_{0}^{M} \int_{0}^{N} g(s, t) n(a(s, t)) d s d t .
\end{aligned}
$$

It follows from (2.10) that

$$
\Omega \leq \frac{\int_{0}^{M} \int_{0}^{N} g(s, t) n(a(s, t)) d s d t}{1-\int_{0}^{M} \int_{0}^{N} c(s, t) g(s, t) n^{\prime}(a(s, t)) d s d t} .
$$

Substituting the inequality above into (2.5), we get the explicit estimate (2.2) for $u(x, y)$.

Remark 2.1. By taking $n(x)=x$, the inequality given in Lemma 2.1 reduces to the inequality given in Lemma 1.1.

Corollary 2.1. Suppose that the conditions of Lemma 2.1 hold. Then

$$
u(x, y) \leq a(x, y)+c(x, y) \int_{0}^{M} \int_{0}^{N} g(s, t) \arctan (u(s, t)) d s d t .
$$

Implies

$$
u(x, y) \leq a(x, y)+\frac{c(x, y) \int_{0}^{M} \int_{0}^{N} g(s, t) \arctan (a(s, t)) d s d t}{1-\int_{0}^{M} \int_{0}^{N} \frac{c(s, t) g(s, t)}{1+a^{2}(s, t)} d s d t},
$$

for $(x, y) \in \Delta$, provided that

$$
\int_{0}^{M} \int_{0}^{N} \frac{c(s, t) g(s, t)}{1+a^{2}(s, t)} d s d t<1,
$$

and if

$$
u(x, y) \leq a(x, y)+c(x, y) \int_{0}^{M} \int_{0}^{N} g(s, t) \ln (u(s, t)+1) d s d t,
$$


then

$$
u(x, y) \leq a(x, y)+\frac{c(x, y) \int_{0}^{M} \int_{0}^{N} g(s, t) \ln (a(s, t)+1) d s d t}{1-\int_{0}^{M} \int_{0}^{N} \frac{c(s, t) g(s, t)}{1+a(s, t)} d s d t}
$$

for $(x, y) \in \Delta$, provided that

$$
\int_{0}^{M} \int_{0}^{N} \frac{c(s, t) g(s, t)}{1+a(s, t)} d s d t<1
$$

Theorem 2.1. Assume that $a(x, y), b(x, y), c(x, y), f(x, y), g(x, y) \in C\left(\Delta, \mathbb{R}_{+}\right)$ and $a(x, y), b(x, y), c(x, y)$ are nondecreasing in $x$ and $y$. Let $n: \mathbb{R}_{+} \rightarrow \mathbb{R}_{+}$is a differentiable increasing function on $] 0,+\infty[$ with continuous non-increasing first derivative $n^{\prime}$ on $] 0,+\infty\left[\right.$. If $u(x, y) \in C\left(\Delta, \mathbb{R}_{+}\right)$satisfies

$u(x, y) \leq a(x, y)+b(x, y) \int_{0}^{x} \int_{0}^{y} f(s, t) u(s, t) d s d t+c(x, y) \int_{0}^{M} \int_{0}^{N} g(s, t) n(u(s, t)) d s d t$,

then, we have

$$
\begin{aligned}
u(x, y) \leq & A^{*}(x, y)+C^{*}(x, y) \times \\
& \frac{\int_{0}^{M} \int_{0}^{N} g(s, t) n\left(A^{*}(s, t)\right) d s d t}{1-\int_{0}^{M} \int_{0}^{N} C^{*}(s, t) g(s, t) n^{\prime}\left(A^{*}(s, t)\right) d s d t}
\end{aligned}
$$

for $(x, y) \in \Delta$, provided that

$$
\int_{0}^{M} \int_{0}^{N} C^{*}(s, t) g(s, t) n^{\prime}\left(A^{*}(s, t)\right) d s d t<1
$$

where

$$
\begin{aligned}
& A^{*}(x, y)=a(x, y) \exp \left\{b(x, y) \int_{0}^{x} \int_{0}^{y} f(s, t) d s d t\right\}, \\
& C^{*}(x, y)=c(x, y) \exp \left\{b(x, y) \int_{0}^{x} \int_{0}^{y} f(s, t) d s d t\right\} .
\end{aligned}
$$

Proof. Fixing any arbitrary $(X, Y) \in \Delta$, then for $(x, y) \in \Delta_{1}=[0, X] \times[0, Y]$, from (2.12), we have

$$
u(x, y) \leq a(X, Y)+b(X, Y) \int_{0}^{x} \int_{0}^{y} f(s, t) u(s, t) d s d t+c(X, Y) \int_{0}^{M} \int_{0}^{N} g(s, t) n(u(s, t)) d s d t
$$

where we apply that $a(x, y), b(x, y)$, and $c(x, y)$ are nondecreasing in $x$ and $y$. 
Define a function $v(x, y),(x, y) \in \Delta_{1}$ by the right side of (2.15). Then, $v(x, y)$ is positive and nondecreasing in $x$ and $y$ and

$$
u(x, y) \leq v(x, y) .
$$

Furthermore, we have

$$
\begin{aligned}
v(0, y)=a(X, Y) & +c(X, Y) \int_{0}^{M} \int_{0}^{N} g(s, t) n(u(s, t)) d s d t \\
\frac{\partial}{\partial x} v(x, y) & =b(X, Y) \int_{0}^{y} f(x, t) u(x, t) d t \\
& \leq b(X, Y) \int_{0}^{y} f(x, t) v(x, t) d t \\
& \leq\left(b(X, Y) \int_{0}^{y} f(x, t) d t\right) v(x, y) .
\end{aligned}
$$

Since $v(x, y)$ is nondecreasing in $y$, from (2.18), one gets

$$
\frac{(\partial / \partial x) v(x, y)}{v(x, y)} \leq b(X, Y) \int_{0}^{y} f(x, t) d t .
$$

Now, keeping $y$ fixed in (2.19), setting $x=s$, and integrating the last inequality with respect to $s$ from 0 to $x$, we get

$$
v(x, y) \leq v(0, y) \exp \left\{b(X, Y) \int_{0}^{x} \int_{0}^{y} f(s, t) d s d t\right\} .
$$

It follows from (2.16) and (2.17) that

$$
\begin{aligned}
u(x, y) \leq & {\left[a(X, Y)+c(X, Y) \int_{0}^{M} \int_{0}^{N} g(s, t) n(u(s, t)) d s d t\right] } \\
& \times \exp \left\{b(X, Y) \int_{0}^{x} \int_{0}^{y} f(s, t) d s d t\right\} \\
= & a(X, Y) \exp \left\{b(X, Y) \int_{0}^{x} \int_{0}^{y} f(s, t) d s d t\right\} \\
& +c(X, Y) \exp \left\{b(X, Y) \int_{0}^{x} \int_{0}^{y} f(s, t) d s d t\right\} \\
& \times \int_{0}^{M} \int_{0}^{N} g(s, t) n(u(s, t)) d s d t \\
= & A_{1}(x, y, X, Y)+C_{1}(x, y, X, Y) \int_{0}^{M} \int_{0}^{N} g(s, t) n(u(s, t)) d s d t
\end{aligned}
$$


where

$$
\begin{aligned}
& A_{1}(x, y, X, Y)=a(X, Y) \exp \left\{b(X, Y) \int_{0}^{x} \int_{0}^{y} f(s, t) d s d t\right\}, \\
& C_{1}(x, y, X, Y)=c(X, Y) \exp \left\{b(X, Y) \int_{0}^{x} \int_{0}^{y} f(s, t) d s d t\right\} .
\end{aligned}
$$

Using Lemma 2.1, from (2.21), we easily obtain

$$
\begin{aligned}
u(x, y) \leq & A_{1}(x, y, X, Y)+C_{1}(x, y, X, Y) \\
& \times \frac{\int_{0}^{M} \int_{0}^{N} g(s, t) n\left(A_{1}(s, t, X, Y)\right) d s d t}{1-\int_{0}^{M} \int_{0}^{N} C_{1}(s, t, X, Y) g(s, t) n^{\prime}\left(A_{1}(s, t, X, Y)\right) d s d t}
\end{aligned}
$$

since the inequality (2.23) holds for all $(x, y) \in \Delta_{1}$, taking $x=X$ and $y=Y$, we have

$$
\begin{aligned}
u(X, Y) \leq & A_{1}(X, Y, X, Y)+C_{1}(X, Y, X, Y) \times \\
& \frac{\int_{0}^{M} \int_{0}^{N} g(s, t) n\left(A_{1}(s, t, X, Y)\right) d s d t}{1-\int_{0}^{M} \int_{0}^{N} C_{1}(s, t, X, Y) g(s, t) n^{\prime}\left(A_{1}(s, t, X, Y)\right) d s d t} \\
= & A^{*}(X, Y)+C^{*}(X, Y) \times \\
& \frac{\int_{0}^{M} \int_{0}^{N} g(s, t) n\left(A^{*}(s, t)\right) d s d t}{1-\int_{0}^{M} \int_{0}^{N} C^{*}(s, t) g(s, t) n^{\prime}\left(A^{*}(s, t)\right) d s d t},
\end{aligned}
$$

for $(X, Y) \in \Delta$, where $A^{*}(X, Y)$ and $C^{*}(X, Y)$ are defined as in (2.14).

Taking into account that $X$ and $Y$ are arbitrary, we replace $X$ and $Y$ by $x$ and $y$, respectively, and we get the required inequality in (2.13).

Remark 2.2. If we take $n(x)=x$, then Theorem 2.1 reduces to Theorem 2 in [5].

Theorem 2.2. Let $a(x, y), b(x, y), c(x, y), f(x, y)$ and $g(x, y)$ be as in Theorem 2.1. If $u(x, y) \in C\left(\Delta, \mathbb{R}_{+}\right)$satisfies

$u^{p}(x, y) \leq a(x, y)+b(x, y) \int_{0}^{x} \int_{0}^{y} f(s, t) u^{q}(s, t) d s d t+c(x, y) \int_{0}^{M} \int_{0}^{N} g(s, t) n(u(s, t)) d s d t$,

where $p \geq q \geq 0, p \geq 1$ are constants, then

$$
\begin{aligned}
u(x, y) \leq & A^{*}(x, y)+C^{*}(x, y) \times \\
& \frac{\int_{0}^{M} \int_{0}^{N} G^{*}(s, t) n\left(A^{*}(s, t)\right) d s d t}{1-\int_{0}^{M} \int_{0}^{N} C^{*}(s, t) G^{*}(s, t) n^{\prime}\left(A^{*}(s, t)\right) d s d t},
\end{aligned}
$$


for $(x, y) \in \Delta$, provided that

$$
\int_{0}^{M} \int_{0}^{N} C^{*}(s, t) G^{*}(s, t) n^{\prime}\left(A^{*}(s, t)\right) d s d t<1,
$$

where

$$
\begin{aligned}
& A^{*}(x, y)=A_{1}(x, y) \exp \left\{B_{1}(x, y) \int_{0}^{x} \int_{0}^{y} F^{*}(s, t) d s d t\right\} \\
& C^{*}(x, y)=C_{1}(x, y) \exp \left\{B_{1}(x, y) \int_{0}^{x} \int_{0}^{y} F^{*}(s, t) d s d t\right\},
\end{aligned}
$$

and

$$
\begin{aligned}
A_{1}(x, y)= & \frac{1}{p} K^{\frac{1-p}{p}} b(x, y) \int_{0}^{x} \int_{0}^{y} f(s, t)\left[\frac{q}{p} K^{(q-p) / p} a(s, t)+\frac{p-q}{p} K^{q / p}\right] d s d t \\
& +\frac{1}{p} K^{\frac{1-p}{p}} a(x, y)+\frac{p-1}{p} K^{\frac{1}{p}} \\
& B_{1}(x, y)=\frac{q}{p} K^{(q-p) / p} b(x, y), C_{1}(x, y)=\frac{1}{p} K^{\frac{1-p}{p}} c(x, y),
\end{aligned}
$$

$$
\begin{aligned}
& F^{*}(x, y)=f(x, y), \\
& G^{*}(x, y)=g(x, y),
\end{aligned}
$$

Proof. Define a function $w(x, y)$ by

$$
\begin{aligned}
w(x, y)= & b(x, y) \int_{0}^{x} \int_{0}^{y} f(s, t) u^{q}(s, t) d s d t \\
& +c(x, y) \int_{0}^{M} \int_{0}^{N} g(s, t) n(u(s, t)) d s d t,
\end{aligned}
$$

for $(x, y) \in \Delta$. Then, from (2.29), we have

$$
u^{p}(x, y) \leq a(x, y)+w(x, y) .
$$

Applying Lemma 1.2, we get

$$
\begin{aligned}
u(x, y) & \leq(a(x, y)+w(x, y))^{1 / p} \leq \frac{1}{p} K^{\frac{1-p}{p}}(a(x, y)+w(x, y))+\frac{p-1}{p} K^{\frac{1}{p}}=v(x, y) . \\
u^{q}(x, y) & \leq(a(x, y)+w(x, y))^{q / p} \leq \frac{q}{p} K^{(q-p) / p}(a(x, y)+w(x, y))+\frac{p-q}{p} K^{q / p} .
\end{aligned}
$$


It follows from $(2.29),(2.30)$ and (2.31) that

$$
\begin{aligned}
w(x, y) \leq & b(x, y) \int_{0}^{x} \int_{0}^{y} f(s, t) \\
& \times\left[\frac{q}{p} K^{(q-p) / p}(a(s, t)+w(s, t))+\frac{p-q}{p} K^{q / p}\right] d s d t \\
& +c(x, y) \int_{0}^{M} \int_{0}^{N} g(s, t) n(v(s, t)) d s d t
\end{aligned}
$$

taking into-account that $\frac{1}{p} K^{\frac{1-p}{p}} w(x, y) \leq v(x, y)$, we have

$$
\begin{aligned}
w(x, y) \leq & b(x, y) \int_{0}^{x} \int_{0}^{y} f(s, t)\left[\frac{q}{p} K^{(q-p) / p} a(s, t)+\frac{p-q}{p} K^{q / p}\right] d s d t \\
& +q K^{(q-1) / p} b(x, y) \int_{0}^{x} \int_{0}^{y} f(s, t) v(s, t) d s d t \\
& +c(x, y) \int_{0}^{M} \int_{0}^{N} g(s, t) n(v(s, t)) d s d t .
\end{aligned}
$$

Multiplying both sides of (2.33) by $\frac{1}{p} K^{\frac{1-p}{p}}$ and adding $\frac{1}{p} K^{\frac{1-p}{p}} a(x, y)+\frac{p-1}{p} K^{\frac{1}{p}}$ to both sides of the resultant inequality, we obtain

$$
\begin{aligned}
v(x, y) \leq & A_{1}(x, y)+B_{1}(x, y) \int_{0}^{x} \int_{0}^{y} F^{*}(s, t) v(s, t) d s d t \\
& +C_{1}(x, y) \int_{0}^{M} \int_{0}^{N} G^{*}(s, t) n(v(s, t)) d s d t,
\end{aligned}
$$

where $A_{1}(x, y), B_{1}(x, y), C_{1}(x, y), F^{*}(x, y)$ and $G^{*}(x, y)$ are defined as in (2.28).

Note that $A_{1}(x, y), B_{1}(x, y)$ and $C_{1}(x, y)$ are nonnegative, continuous, and nondecreasing for $(x, y) \in \Delta$.A suitable application of Theorem 2.1 to (2.34) gives

$$
\begin{aligned}
u(x, y) \leq & v(x, y) \leq A^{*}(x, y)+C^{*}(x, y) \times \\
& \frac{\int_{0}^{M} \int_{0}^{N} G^{*}(s, t) n\left(A^{*}(s, t)\right) d s d t}{1-\int_{0}^{M} \int_{0}^{N} C^{*}(s, t) G^{*}(s, t) n^{\prime}\left(A^{*}(s, t)\right) d s d t},
\end{aligned}
$$

where $A^{*}(x, y)$ and $C^{*}(x, y)$ are defined as in (2.27).

Remark 2.3. If we take $n(x)=x$, then Theorem 2.2 reduces to Theorem 6 in [5] .

\section{Applications}

In this section, we shall illustrate how our main results can be applied to study the boundedness and uniqueness of the solution to certain integral equations in two independent variables. 
Example 3.1. Consider the following integral equation:

$$
z(x, y)=a(x, y)+b(x, y) \int_{0}^{x} \int_{0}^{y} F(s, t, z) d s d t+c(x, y) \int_{0}^{M} \int_{0}^{N} G(s, t, z) d s d t
$$

for $(x, y) \in \Delta$, where $z(x, y) \in C(\Delta, \mathbb{R}), a(x, y), b(x, y), c(x, y) \in C\left(\Delta, \mathbb{R}_{+}\right)$are nondecreasing in $x$ and $y, F(x, y, z), G(x, y, z) \in C(\Delta \times \mathbb{R}, \mathbb{R})$.

Theorem 3.1. Assume that the functions $F$ and $G$ in (3.1) satisfy the conditions

$$
\begin{aligned}
|F(s, t, z)| & \leq f(s, t)|z| \\
|G(s, t, z)| & \leq g(s, t) n(|z|),
\end{aligned}
$$

where $f(s, t), g(s, t)$ and $n$ are defined as in Theorem 2.1.

If $z(x, y)$ is the unique solution of (3.1), then

$$
\begin{aligned}
|z(x, y)| \leq & A^{*}(x, y)+C^{*}(x, y) \times \\
& \frac{\int_{0}^{M} \int_{0}^{N} g(s, t) n\left(A^{*}(s, t)\right) d s d t}{1-\int_{0}^{M} \int_{0}^{N} C^{*}(s, t) g(s, t) n^{\prime}\left(A^{*}(s, t)\right) d s d t},
\end{aligned}
$$

for $(x, y) \in \Delta$, provided that

$$
\int_{0}^{M} \int_{0}^{N} C^{*}(s, t) g(s, t) n^{\prime}\left(A^{*}(s, t)\right) d s d t<1
$$

where $A^{*}(x, y), C^{*}(x, y)$ are defined in (2.14).

Proof. Assume that $z(x, y)$ is the unique solution of (3.1), from (3.2) we have

$$
\begin{aligned}
|z(x, y)| \leq & a(x, y)+b(x, y) \int_{0}^{x} \int_{0}^{y} f(s, t)|z(s, t)| d s d t \\
& +c(x, y) \int_{0}^{M} \int_{0}^{N} g(s, t) n(|z(s, t)|) d s d t .
\end{aligned}
$$

Now an application of Theorem 2.1 to (3.5), yields the required inequality in $(3.3)$.

Corollary 3.1. If we take in (3.2), $n(z)=\arctan (z)$, then the unique solution of (3.1) can be expressed as

$$
\begin{aligned}
|z(x, y)| \leq & A^{*}(x, y)+C^{*}(x, y) \times \\
& \frac{\int_{0}^{M} \int_{0}^{N} g(s, t) \arctan \left(A^{*}(s, t)\right) d s d t}{1-\int_{0}^{M} \int_{0}^{N} \frac{C^{*}(s, t) g(s, t) d s d t}{1+A^{* 2}(s, t)}}
\end{aligned}
$$


provided that

$$
\int_{0}^{M} \int_{0}^{N} \frac{C^{*}(s, t) g(s, t) d s d t}{1+A^{* 2}(s, t)}<1 .
$$

If we take $n(z)=\ln (z+1)$, then the unique solution of (3.1) can be expressed as

$$
\begin{aligned}
|z(x, y)| \leq & A^{*}(x, y)+C^{*}(x, y) \times \\
& \frac{\int_{0}^{M} \int_{0}^{N} g(s, t) \ln \left(A^{*}(s, t)+1\right) d s d t}{1-\int_{0}^{M} \int_{0}^{N} \frac{C^{*}(s, t) g(s, t) d s d t}{1+A^{*}(s, t)}}
\end{aligned}
$$

provided that

$$
\int_{0}^{M} \int_{0}^{N} \frac{C^{*}(s, t) g(s, t) d s d t}{1+A^{*}(s, t)}<1 .
$$

Proposition 3.1. Assume that the functions $F$ and $G$ in (3.1) satisfy the conditions

$$
\begin{aligned}
|F(s, t, z)|-F(s, t, \bar{z}) & \leq f(s, t)|z-\bar{z}|, \\
|G(s, t, z)|-G(s, t, \bar{z}) & \leq g(s, t) n(|z-\bar{z}|),
\end{aligned}
$$

where $f(s, t), g(s, t)$ and $n$ are defined as in Theorem 2.1 with $n(0)=0$. If

$$
\int_{0}^{M} \int_{0}^{N} C^{*}(s, t) g(s, t) n^{\prime}\left(A^{*}(s, t)\right) d s d t<1,
$$

where $A^{*}$ and $C^{*}$ are defined as in Theorem 2.1, and $z(x, y)$ is a solution of (3.1), then (3.1) has at most one solution.

Proof. Let $z(x, y)$ and $\bar{z}(x, y)$ be two solutions of (3.1), then

$$
\begin{aligned}
\bar{z}(x, y)= & a(x, y)+b(x, y) \int_{0}^{x} \int_{0}^{y} F(s, t, \bar{z}) d s d t \\
& +c(x, y) \int_{0}^{M} \int_{0}^{N} G(s, t, \bar{z}) d s d t, \\
z(x, y)= & a(x, y)+b(x, y) \int_{0}^{x} \int_{0}^{y} F(s, t, z) d s d t \\
& +c(x, y) \int_{0}^{M} \int_{0}^{N} G(s, t, z) d s d t .
\end{aligned}
$$


From (3.7), we have

$$
\begin{aligned}
|z(x, y)-\bar{z}(x, y)| \leq & b(x, y) \int_{0}^{x} \int_{0}^{y}|F(s, t, z)-F(s, t, \bar{z})| d s d t \\
& +c(x, y) \int_{0}^{M} \int_{0}^{N}|G(s, t, z)-G(s, t, \bar{z})| d s d t \\
\leq & b(x, y) \int_{0}^{x} \int_{0}^{y} f(s, t)|z-\bar{z}| d s d t \\
+ & c(x, y) \int_{0}^{M} \int_{0}^{N} g(s, t) n(|z-\bar{z}|) d s d t .
\end{aligned}
$$

According to Theorem 2.1, we obtain that $|z(x, y)-\bar{z}(x, y)| \leq 0$, which implies $z(x, y)=\bar{z}(x, y)$ for $(x, y) \in \Delta$.

\section{R E F E R E N C E S}

1. D. Bainov and P. Simeonov, Integral inequalities and applications, vol 57 of Mathematics and its applications, Kluwer Academic Publishers, Dordrecht, the netherlands, 1992.

2. B.Ben Nasser, K. Boukerrioua and M. A. Hammami," On the stability of perturbed time scale systems using integral inequalities. Applied Sciences, 16, 56 - 71 .

3. B.Ben Nasser, K. Boukerrioua and M. A. Hammami," On stability and stabilization of perturbed time scale systems with Gronwall inequalities". Journal of Mathematical Physics, Analysis, Geometry, 11(3), $207-235$.

4. K. Boukerrioua and A.Guezane-Lakoud, Some nonlinear integral inequalities arising in differential equations, EJDE, Vol 2008 , No.80, pp.1 - 6. http://ejde.math.txstate.

5. K. Cheng, C. Guo, New Explicit Bounds on Gamidov Type Integral Inequalities for Functions in Two Variables and Their Applications, Abstract and Applied Analysis , 2014, Article ID 539701, 9 pages.

6. K. Cheng, C. Guo, M. Tang, Some Nonlinear Gronwall-Bellman-Gamidov Integral Inequalities and Their Weakly Singular Analogues with Applications, Abstract and Applied Analysis, 2014, Article ID 562691,9 pages.

7. S. S. Dragomir and Y.-H. Kim, "Some integral inequalities for functions of two variables," Electronic Journal of Differential Equations, vol. 2003, article 10, 2003.

8. Sh. G. Gamidov, "Certain integral inequalities for boundary value problems of differential equations", Differ. Uravn., 5:3 (1969), 463472.

Differential Equations, vol. 5,pp.463 - 472, 1969.

9. F.Jiang, F. Meng, "Explicit bounds on some new nonlinear integral inequalities with delay". Journal of Computational and Applied Mathematics, 205(2007), 479 - 486.

10. S. D.Kendre, S. G. Latpate, On some mixed integral inequalities and its applications. Theoretical Mathematics \& Applications, vol.5(2013), no.1, 1 - 14 .

11. M. Medved, "Nonlinear singular integral inequalities for functions in two and $n$ independent variables," Journal of Inequalities and Applications, vol. 5, no. 3, pp. $287-308,2000$. 
12. B. G. Pachpatte, "A note on certain integral inequality," Tamkang Journal of Mathematics, vol. 33 , no. 4 , pp. $353-358,2002$.

13. B.G.Pachpatte, "Integral and finite difference inequalities and applications," NorthHolland Mathematics studies, 205, 2006.

14. B. G. Pachpatte, "Explicit bounds on Gamidov type integral inequalities," Tamkang Journal of Mathematics, vol. 37, no. 1, pp.1 - 9, 2006.

15. Y. Tian, M. Fan, and F. Meng, "A generalization of retarded integral inequalities in two independent variables and their applications," Applied Mathematics and Computation, vol. 221,pp.239 - 248, 2013.

16. W.-S. Wang, "A generalized retarded Gronwall-like inequality in two variables and applications to BVP," Applied Mathematics and Computation, vol. 191, no. 1, pp. $144-154,2007$.

17. W.-S. Wang and C.-X. Shen, "On a generalized retarded integral inequality with two variables," Journal of Inequalities and Applications, vol. 2008, Article ID 518646, 2008.

18. H. Zhang and F. Meng, "On certain integral inequalities in two independent variables for retarded equations," Applied Mathematics and Computation, vol. 203, no. 2, pp. $608-616,2008$.

19. K. Zheng, "Some retarded nonlinear integral inequalities in two variables and applications," Journal of Inequalities in Pure and Applied Mathematics, vol. 9, no. 2, article 57,2008 .

Khaled Boukerrioua

Lanos Laboratory

Department of Mathematics

Faculty of Sciences

Badji-Mokhtar University, BP 12, Annaba, Algeria

khaledv2004@yahoo.fr

Dallel Diabi

Lanos Laboratory

Department of Mathematics

Faculty of Sciences

Badji-Mokhtar University, BP 12, Annaba, Algeria

dalleldiabi@yahoo.fr

Brahim Kilani

Department of Mathematics

Faculty of Sciences

Badji-Mokhtar University, BP 12, Annaba, Algeria

kilbra2000@yahoo.fr 